\title{
Poly(lactic-co-glycolic) Acid-Chitosan Dual Loaded Nanoparticles for Antiretroviral Nanoformulations
}

\author{
Faithful Makita-Chingombe, ${ }^{1}$ Hilliard L. Kutscher, ${ }^{2,3}$ Sara L. DiTursi, ${ }^{4}$ \\ Gene D. Morse, ${ }^{3,4}$ and Charles C. Maponga ${ }^{1,3,4}$ \\ ${ }^{1}$ School of Pharmacy, University of Zimbabwe, P.O. Box MP167, Mount Pleasant, Harare, Zimbabwe \\ ${ }^{2}$ The Institute of Lasers, Photonics and Biophotonics, University at Buffalo, Buffalo, NY 14260, USA \\ ${ }^{3}$ Center for Integrated Global Health Sciences, Translational Pharmacology Research Core, New York State Center of Excellence in \\ Bioinformatics and Life Sciences, University at Buffalo, Buffalo, NY 14203, USA \\ ${ }^{4}$ School of Pharmacy and Pharmaceutical Sciences, University at Buffalo, Buffalo, NY 14214, USA
}

Correspondence should be addressed to Faithful Makita-Chingombe; faithmakita@gmail.com

Received 16 January 2016; Revised 11 March 2016; Accepted 20 March 2016

Academic Editor: Philippe Maincent

Copyright (c) 2016 Faithful Makita-Chingombe et al. This is an open access article distributed under the Creative Commons Attribution License, which permits unrestricted use, distribution, and reproduction in any medium, provided the original work is properly cited.

\begin{abstract}
Poly(lactic-co-glycolic acid) (PLGA) chitosan (CS) coated nanoparticles (NPs) were loaded with two antiretrovirals (ARVs) either lamivudine (LMV) which is hydrophilic or nevirapine (NVP) which is hydrophobic or both LMV and NVP. These ARVs are of importance in resource-limited settings, where they are commonly used in human immunodeficiency virus (HIV-1) treatment due to affordability and accessibility. NPs prepared by a water-oil-water emulsion and reduced pressure solvent evaporation technique were determined to have a positive zeta potential, a capsule-like morphology, and an average hydrodynamic diameter of $240 \mathrm{~nm}$. Entrapment of NVP as a single ARV had a notable increase in NP size compared to LMV alone or in combination with LMV. NPs stored at room temperature in distilled water maintained size, polydispersity (PDI), and zeta potential for one year. No changes in size, PDI, and zeta potential were observed for NPs in $10 \%$ sucrose in lyophilized or nonlyophilized states stored at $4{ }^{\circ} \mathrm{C}$ and $-20^{\circ} \mathrm{C}$, respectively. Freezing NPs in the absence of sucrose increased NP size. Drug loading, encapsulation efficiency, and kinetic release profiles were quantified by high performance liquid chromatography (HPLC). Our novel nanoformulations have the potential to improve patient outcomes and expand drug access in resource-limited countries for the treatment of HIV-1.
\end{abstract}

\section{Introduction}

HIV infection is one of the deadliest diseases worldwide, particularly in resource-limited settings. Antiretrovirals (ARVs) have offered life-sustaining treatment for people living with HIV infection and acquired immunodeficiency syndrome (AIDS). As of March 2015, ARV access remains limited with only 15 million ( 40\%) of the 36.9 million individuals infected worldwide receiving treatment $[1,2]$. Combination antiretroviral therapy (cART) is highly effective because it targets multiple stages of the HIV lifecycle. While patient access to ARVs is increasing, adverse effects, large pill burden, and frequent administration of many first generation ARVs have led to their reduced use. This provides an opportunity to reformulate currently approved cART therapies.

The World Health Organization (WHO) guidelines for the use of ARVs in treating HIV infection in adults recommend NVP in combination with zidovudine/lamivudine or tenofovir/lamivudine when combination therapy with tenofovir/lamivudine/efavirenz is contraindicated or not available [3]. NVP and LMV are still utilized as first-line ARVs particularly in resource-limited settings due to their availability and lower cost. Although more efficacious regimens have been recommended by the WHO, their availability has been largely delayed in resource-limited settings. Additional barriers also exist that make adherence to the prescribed 
regimen a challenge for patients being treated for HIV such as stigma, complexity of regimens, pill burden/fatigue, food requirements, adverse effects, nondisclosure, failure to fill prescriptions, and cost-related issues [4]. Therefore, longacting, cell-targeted ARVs have become a major area of research.

NPs have revolutionized sustained drug delivery and cell specific targeting approaches and have been developed to deliver conventional drugs, recombinant proteins, vaccines, and nucleotides. Modifying plasma exposure through sustained release profiles and/or cell targeting can further reduce the toxicities associated with these therapeutics. Therapeutic agents that are efficacious but have serious adverse effects and/or toxicities have the potential to be reinvestigated and reformulated as NPs in order to diminish or eliminate these unfavorable properties. For example, doxorubicin is an FDA approved chemotherapeutic agent that was reformulated into PEGylated liposomes (Doxil ${ }^{\circledR}$ ). Doxil demonstrated enhanced antitumor efficacy compared to doxorubicin alone and had a lower incidence of toxicities, most notably cardiotoxicity [5]. Paclitaxel, an FDA approved chemotherapeutic, was reformulated into albumin-based NPs (Abraxane ${ }^{\circledR}$ ). Abraxane greatly reduced adverse effects associated with the former formulation [6].

A major concern with reformulation of a drug is maintaining its stability during manufacturing and storage. Biodegradable and biocompatible polymers such as poly(lactic-co-glycolic acid) (PLGA) have been shown to protect drug molecules from enzymatic degradation and provide physicochemical stability [7].

NPs can be optimized by size and shape or functionalized with protein and lipid coatings to facilitate their drug release, cellular uptake, and ability to cross physiologic barriers, for example, the blood-brain barrier [8-10]. Furthermore, NPs can be functionalized with ligands such as those with immune system modulating effects, concurrently modifying the cellular immune response and enhancing intracellular drug delivery [11]. Chitosan (CS) has gained attention in the nanomedicine field because it carries a positive charge that can be utilized for cellular and anatomic targeting of NPs [12]. The electrostatic interactions between positively charged CS NPs and the negatively charged cell surface have been shown to enhance nanoparticle uptake [13-18]. By using a PLGA core in conjunction with a CS shell, both hydrophobic and hydrophilic drugs can be encapsulated within NP.

The objective of this study was to prepare and characterize NVP- and LMV-NPs both as single ARVs and in combination and to assess their physicochemical stability.

\section{Materials and Methods}

2.1. Materials. Poly(lactic-co-glycolic acid) (PLGA) (lactide : glycolide $=50: 50$, molecular weight $(\mathrm{MW})=38-$ $54 \mathrm{kDa})$, poly(vinyl alcohol) (PVA) (Mowiol ${ }^{\circledR} 4-88, \mathrm{MW}=$ $31 \mathrm{kDa})$, and chitosan $(\mathrm{CS})$ oligosaccharide lactate $(\mathrm{MW}=$ $5 \mathrm{kDa}$ ) were purchased from Sigma Aldrich (St. Louis, MO). Nevirapine (NVP) and lamivudine (LMV) were purchased from TCI America (Portland, OR). All other reagents were used as received without further purification.
2.2. Preparation of NPs. NPs were prepared using a modified water-oil-water $(\mathrm{w} / \mathrm{o} / \mathrm{w})$ double emulsion and solvent evaporation technique [19]. LMV, NVP, or both (4 mg) were added to a microcentrifuge tube containing $200 \mu \mathrm{L}$ water with $90 \mu \mathrm{g}$ of CS and $500 \mu \mathrm{L}$ of $10 \mathrm{mg} / \mathrm{mL}$ PLGA dissolved in dichloromethane (DCM). The microcentrifuge tube was probe sonicated (750-watt probe sonicator, 1/4-inch probe tip, Cole-Parmer Vernon Hills, IL) at 21\% amplitude for 30 seconds in a $4^{\circ} \mathrm{C}$ water bath. The resulting emulsion was quickly added to $2 \mathrm{~mL}$ of $2.5 \%$ (w/v) PVA in water in a $5 \mathrm{~mL}$ centrifuge tube and probe sonicated again for 90 seconds in a $4^{\circ} \mathrm{C}$ water bath to obtain the w/o/w emulsion. The resulting w/o/w emulsion was quickly added dropwise to $10.25 \mathrm{~mL}$ of $0.025 \%$ PVA in a $100 \mathrm{~mL}$ round bottom flask with a stir bar. Under constant stirring, DCM was evaporated under vacuum. The NP suspension was washed once with water followed by centrifugation at $14,000 \times \mathrm{g}$ for 15 minutes. NPs were resuspended in water or $10 \%(\mathrm{w} / \mathrm{v})$ sucrose prior to storage or characterization.

2.3. Characterization of NPs. NP size and polydispersity index (PDI) were determined by 3 ways: (1) dynamic light scattering (DLS) (Brookhaven 90 plus Particle Analyzer, Brookhaven Instruments, Holtsville, NY) at an angle of $90^{\circ}$; (2) nanoparticle tracking analysis (NTA) (LM-10, Malvern Instruments, Westborough, MA); and (3) transmission electron microscopy (TEM). Surface morphology was analyzed by TEM. TEM samples were prepared by staining with $0.1 \% \mathrm{w} / \mathrm{v}$ phosphotungstic acid. Zeta potential was determined by Zeta PALS (Brookhaven Instruments). ARV loading and in vitro release were characterized by high performance liquid chromatography (HPLC) (Alliance 2795, Waters Corporation, Milford, MA).

2.4. Chromatographic Separation. Detection of NVP and LMV was adapted from Rezk et al. [20]. ARVs were separated by reverse phase chromatography on an Atlantis $\mathrm{dC}_{18}$ column $(3.9 \times 150 \mathrm{~mm}, 5 \mu \mathrm{m}$, Waters Corporation $)$ with an Atlantis $\mathrm{dC}_{18}$ guard column $(3.9 \times 20 \mathrm{~mm}, 5 \mu \mathrm{m}$, Waters Corporation) and detected using a photodiode array detector (PDA 996, Waters Corporation) collecting at $270 \mathrm{~nm}$ for LMV and $282 \mathrm{~nm}$ for NVP. Zalcitabine (ddC) was used as the internal standard (IS) and detected at both 270 and $282 \mathrm{~nm}$. Chromatographic separation was performed with gradient elution. LMV, NVP, and IS eluted at 6.1, 14.1, and 7.7 minutes, respectively. The two mobile phase components were as follows: mobile phase A: $10 \mathrm{mM}$ ammonium acetate; mobile phase B: $200 \mathrm{~mL}$ mobile phase A, $500 \mathrm{~mL}$ acetonitrile, and $300 \mathrm{~mL}$ methanol. Both mobile phases were filtered through a $0.22 \mu \mathrm{m}$ membrane filter (Millipore, Milford, MA). A linear gradient was programmed in which the initial percentages of mobile phases A and B were $96 \%$ and $4 \%$, respectively. Mobile phase $\mathrm{B}$ changed to $10 \%$ at 8 minutes, $30 \%$ at 9.5 minutes, $43 \%$ at 13 minutes, and then to the initial $4 \%$ at 16.5 minutes up to 20 minutes. The mobile phase flow rate was $1 \mathrm{~mL} / \mathrm{minute}$, column temperature was set to $40^{\circ} \mathrm{C}$, and injection volume was $100 \mu \mathrm{L}$. Data were collected and processed using Waters Empower Chromatography Software. The calibration curve 
was linear from $200 \mathrm{ng} / \mathrm{mL}$ to $10,000 \mathrm{ng} / \mathrm{mL}$ for NVP and $50 \mathrm{ng} / \mathrm{mL}$ to $10,000 \mathrm{ng} / \mathrm{mL}$ for LMV.

2.5. ARV Loading and Encapsulation Efficiency. The amounts of NVP and LMV entrapped in the nanoparticles were determined using the HPLC method described above. A $1 \mathrm{~mL}$ aliquot of PLGA-CS NPs was centrifuged for 15 minutes at $14,000 \times \mathrm{g}$. The supernatant was removed and $0.5 \mathrm{~mL}$ acetonitrile and $0.5 \mathrm{~mL}$ water were added to dissolve the PLGA-CS NPs. Each sample was bath sonicated for 5 minutes followed by drying under nitrogen gas (Zymark TurboVap LV, Hopkinton, MA). Samples were then reconstituted with HPLC grade water before measuring ARV content by the HPLC method described above. The percentage of drug loading and encapsulation efficiency were calculated using the following equations:

$$
\begin{aligned}
& \text { Drug loading efficiency } \\
& \qquad=\left(\frac{\text { amount of drug loaded }}{\text { amount of PLGA }}\right) \times 100 \%,
\end{aligned}
$$

Encapsulation efficiency

$$
=\left(\frac{\text { amount of drug loaded }}{\text { total drug amount }}\right) \times 100 \% \text {. }
$$

2.6. ARV Release Kinetics. Release kinetics of dual and singly loaded ARV PLGA-CS NPs were evaluated by taking $1 \mathrm{~mL}$ aliquots of PLGA-CS NPs. Each aliquot was washed twice with distilled water by centrifuging the sample for 15 minutes at $14,000 \times \mathrm{g}$ and removing the supernatant. The pellet was resuspended in $1 \mathrm{~mL}$ of $10 \mathrm{mM}$ phosphate buffer, $\mathrm{pH} 7.2$ or $\mathrm{pH}$, and added to a microcentrifuge tube. Microcentrifuge tubes were stored at $37^{\circ} \mathrm{C}$ or $4^{\circ} \mathrm{C}$. At predetermined time points, a microcentrifuge tube was removed from the storage condition and centrifuged for 15 minutes at $14,000 \times \mathrm{g}$. From the microcentrifuge tube, $250 \mu \mathrm{L}$ of supernatant was aliquoted, spiked with IS, and analyzed for ARV using the HPLC method described above. Data were fit using GraphPad Prism 6.0 (La Jolla, CA) to several drug release models including zero order, first order, Higuchi, Korsmeyer-Peppas, and Hixson-Crowell to determine the release mechanism.

2.7. Stability of NPs. Size, PDI, surface morphology, surface charge (zeta potential), and drug encapsulation were determined at time of fabrication, two weeks, one month, and one year for single ARV or dual ARV-loaded NPs stored at room temperature, $4^{\circ} \mathrm{C},-20^{\circ} \mathrm{C}$, and $-70^{\circ} \mathrm{C}$. The stability of NPs lyophilized with and without $10 \%$ sucrose was also evaluated.

\section{Results and Discussion}

Recently, a paradigm shift in HIV research from drug discovery to drug delivery has occurred [21]. Novel ARV nanoformulations have shown improvements in pharmacokinetics which have the potential to simplify dosing strategies, increase patient adherence, and maximize antiviral efficacy $[22,23]$. Rilpivirine (TMC-278, Edurant ${ }^{\circledR}$ ) and cabotegravir
(GSK1265744) are two ARVs currently in phase II/III trials as once monthly, long-acting injectables. Clinical trials have shown promise for these agents as long-acting NP injections for the treatment of HIV-1 infection. These novel formulations have been shown to maintain plasma concentrations significantly above the target plasma inhibitory concentration, reducing the likelihood of subtherapeutic plasma concentrations and thus reducing the potential for viral resistance. These formulations are administered monthly or even quarterly as opposed to once daily dosing, which can greatly improve patient adherence to ARVs.

We used an existing encapsulation method to load ARVs into polymeric NPs. The emulsion-solvent-evaporation technique is a proven method for encapsulating hydrophobic therapeutics but has poor results in encapsulating hydrophilic therapeutics. This study utilized a modification of this technique by adding an additional emulsification step which provides an opportunity to encapsulate hydrophilic agents $[24,25]$. By fabricating NP with both PLGA and CS, we are able to successfully incorporate two ARVs that have markedly different aqueous solubilities and hydrophobicities (NVP: $0.1 \mathrm{mg} / \mathrm{mL}, \log P=2.5 ; \mathrm{LMV}: 70 \mathrm{mg} / \mathrm{mL}, \log P=-1.4)$.

3.1. Morphology and Size of Single and Dual Loaded NPs. Figure 1 illustrates the capsule morphology of ARV-loaded NPs confirming the existence of an inner core and an outer shell. We postulate that NVP is encapsulated within the hydrophobic inner core of PLGA while LMV is encapsulated within the hydrophilic CS outer shell.

Small-sized NPs are associated with higher uptake by cells, offering an efficient delivery system. However, drug loading efficiency of passively entrapped therapeutics decreases with particle size [16]. To enable sufficient drug loading, we targeted NPs that are $250 \mathrm{~nm}$ in diameter. In this study, NPs had an average diameter of $242 \mathrm{~nm}$ (Table 1) measured by DLS. In DLS, the intensity weighted function of the hydrodynamic diameter is biased to larger NPs [26]. However, when comparing the number-weighted DLS result to NTA and TEM, they are comparable (Table 2). The low polydispersity and size distribution of Sample 3.4 is shown in Figure 2.

NP size was influenced by the hydrophobicity of the encapsulated ARV. PLGA-CS-LMV NPs had an average diameter of $237 \mathrm{~nm}$, whereas PLGA-CS-NVP NPs had an average diameter of $282 \mathrm{~nm}$. The increase in size of the NVP-loaded NPs can be attributed to encapsulation of the hydrophobic drug entrapped in the core leading to changes in the packing structure of the polymer upon NP fabrication. In addition, the physicochemical interactions between PLGA and NVP influenced the increased PDI of the PLGA-CS-NVP NPs compared to PLGA-CS-LMV NPs (Table 1). However, PDI remained below 0.2 , which is considered a monodisperse system [27]. Monodisperse NPs are ideal in drug delivery as they tend to yield more consistent pharmacokinetic properties as a function of their size, for example, release kinetics and biodistribution [28, 29].

NPs formulated with CS exhibited a positive zeta potential suggesting successful coating with CS (Table 1). This 


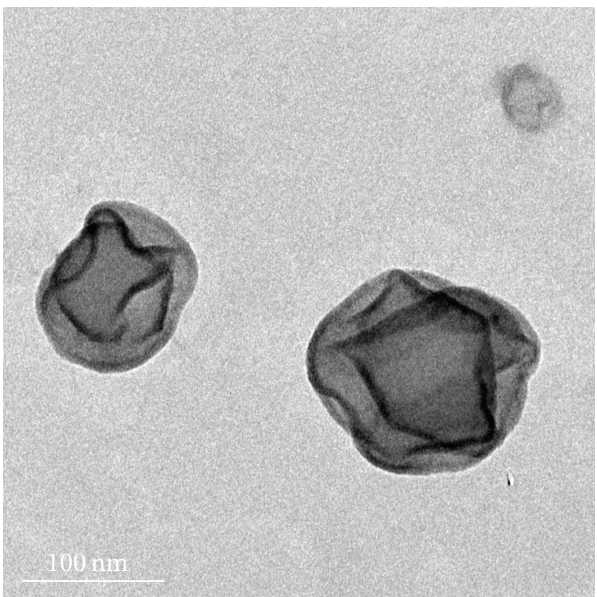

(a)

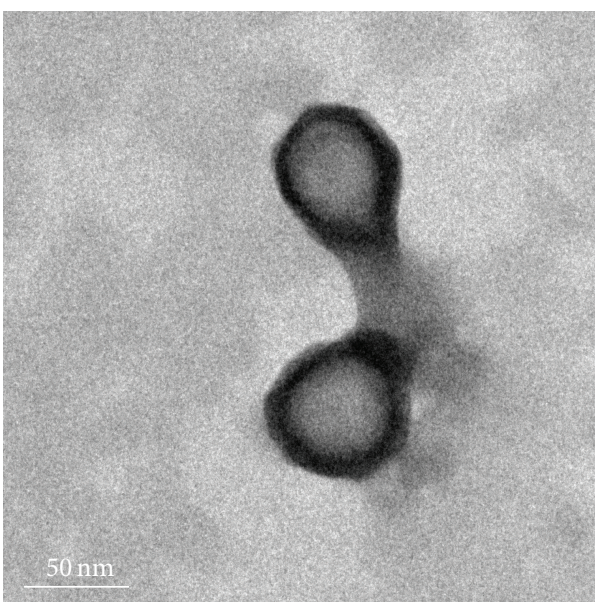

(c)

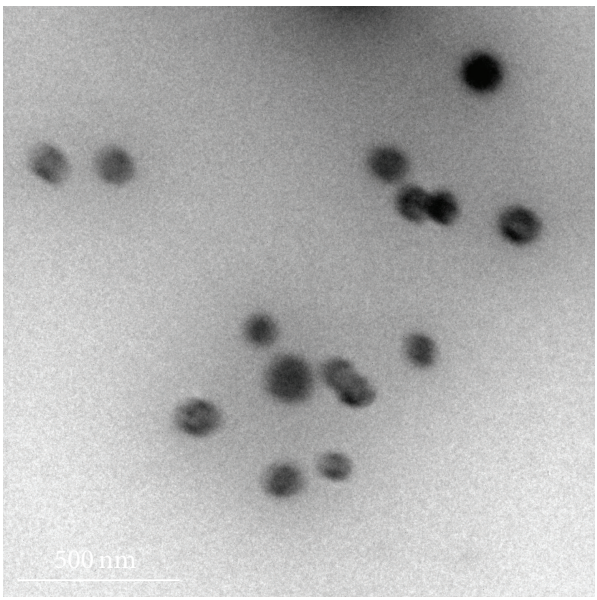

(e)

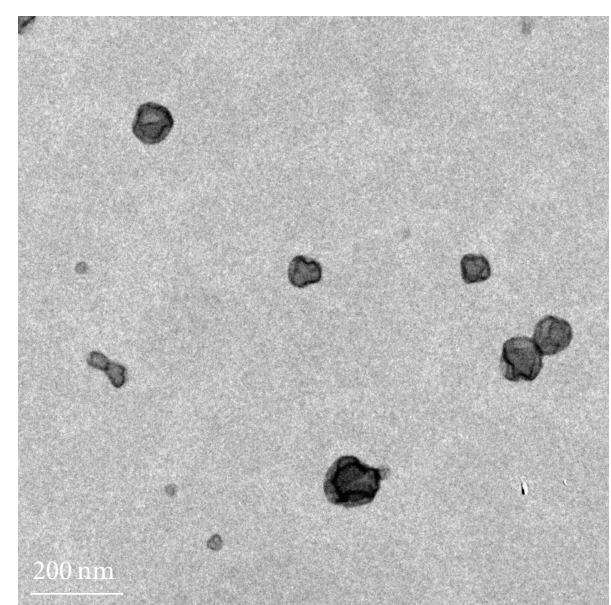

(b)

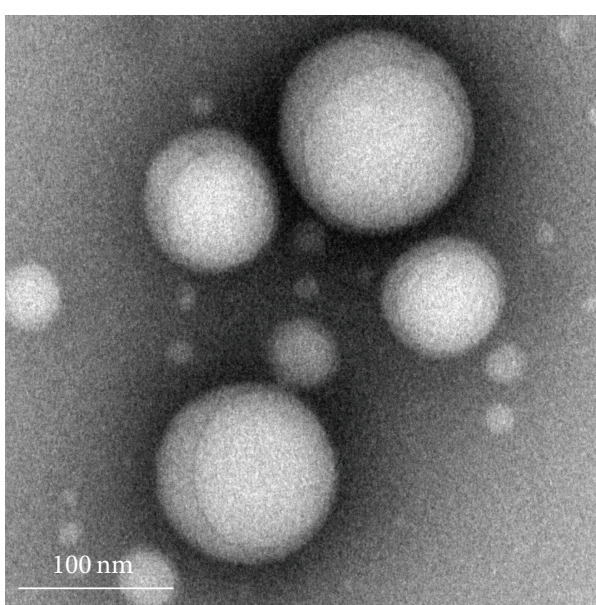

(d)

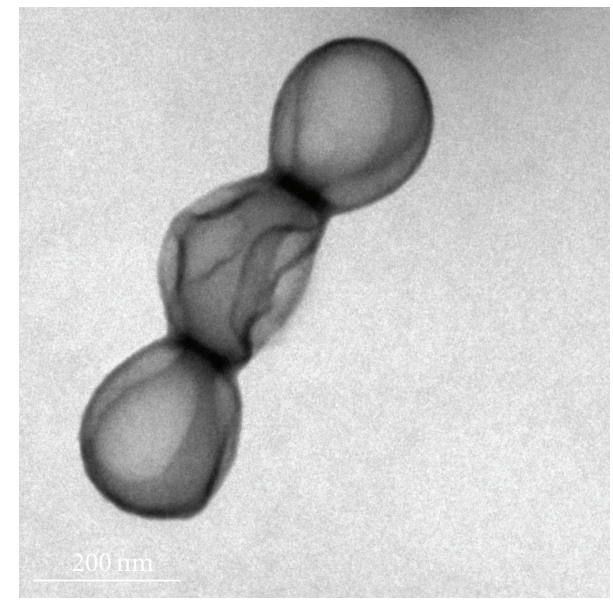

(f)

FIGURE 1: TEM image of PLGA-CS-NVP-LMV NPs. NPs following fabrication ( $a, b)$; after 1 year, stored at room temperature (c); after 1 year, stored at $-70^{\circ} \mathrm{C}(\mathrm{d})$; lyophilized with $10 \%$ sucrose, stored at $4^{\circ} \mathrm{C}(\mathrm{e})$; and stored at $-20^{\circ} \mathrm{C}$ in $10 \%$ sucrose but nonlyophilized (f). 
TABLE 1: Average size, PDI, zeta potential, and encapsulation efficiency of single and dual drug loaded NPs.

\begin{tabular}{|c|c|c|c|c|}
\hline Sample & Components & Size $(\mathrm{nm})$ & Polydispersity index (PDI) & Zeta potential $(\mathrm{mV})$ \\
\hline 1.1 & PLGA-CS-LMV & 233 & 0.084 & +25 \\
\hline 1.2 & PLGA-CS-LMV & 231 & 0.072 & +19 \\
\hline 1.3 & PLGA-CS-LMV & 240 & 0.127 & +28 \\
\hline 1.4 & PLGA-CS-LMV & 244 & 0.148 & +25 \\
\hline Mean & & 237 & 0.108 & +24 \\
\hline Std. Dev. & & 6 & 0.04 & 3.9 \\
\hline Average EE\% & & $7.5 \%$ & & \\
\hline 2.1 & PLGA-CS-NVP & 317 & 0.314 & +18 \\
\hline 2.2 & PLGA-CS-NVP & 250 & 0.245 & +22 \\
\hline 2.3 & PLGA-CS-NVP & 293 & 0.272 & +20 \\
\hline 2.4 & PLGA-CS-NVP & 273 & 0.206 & +22 \\
\hline Mean & & 283 & 0.259 & +20.4 \\
\hline Std. Dev. & & 29 & 0.05 & 2.0 \\
\hline Average EE\% & & $7.5 \%$ & & \\
\hline 3.1 & PLGA-CS-NVP-LMV & 246 & 0.176 & +23 \\
\hline 3.2 & PLGA-CS-NVP-LMV & 242 & 0.131 & +25 \\
\hline 3.3 & PLGA-CS-NVP-LMV & 261 & 0.151 & +15 \\
\hline 3.4 & PLGA-CS-NVP-LMV & 220 & 0.122 & +21 \\
\hline Mean & & 242 & 0.145 & +21 \\
\hline Std. Dev. & & 17 & 0.02 & 4.2 \\
\hline Average EE\% & & $13 \%$ & & \\
\hline 4.1 & PLGA-CS & 227 & 0.010 & +21 \\
\hline 4.2 & PLGA-CS & 242 & 0.118 & +18 \\
\hline Mean & & 235 & 0.064 & +20 \\
\hline Std. Dev. & & 11 & 0.08 & 1.8 \\
\hline 5.1 & PLGA-LMV & 278 & 0.125 & -21 \\
\hline 6.1 & PLGA-NVP & 306 & 0.305 & -9 \\
\hline
\end{tabular}

TABLE 2: Comparison of NPs size determinations by DLS and NTA.

\begin{tabular}{lccc}
\hline \multirow{2}{*}{ Formulation } & \multicolumn{2}{c}{ Dynamic light scattering (DLS) } & \multicolumn{2}{c}{$\begin{array}{c}\text { Nanoparticle size tracking analysis (NTA) } \\
\text { By number }\end{array}$} \\
\hline PLGA-LMV & By intensity & 169 & 169 \\
PLGA-CS-LMV & 279 & 130 & 170 \\
PLGA-CS & 234 & 147 & 156 \\
\hline
\end{tabular}

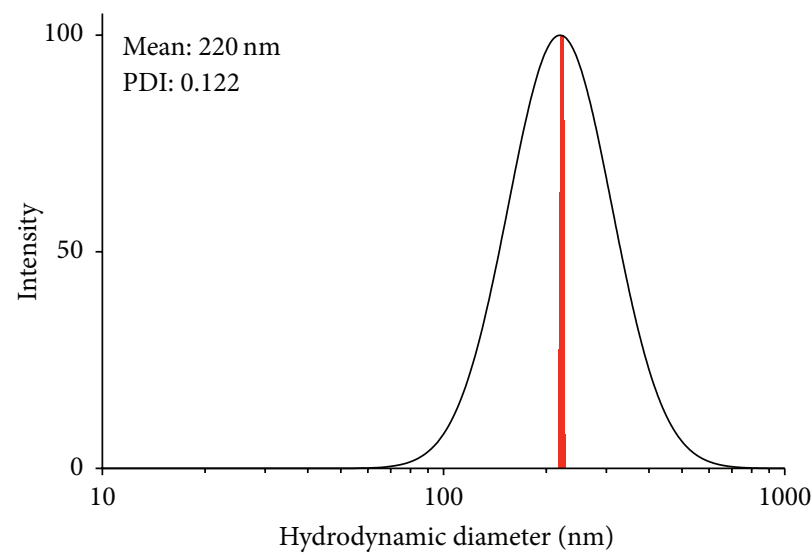

Figure 2: Log-normal distribution for PLGA-CS-NVP-LMV NPs (Sample 3.4). MSD histogram in red. 
overall positive charge is a direct result of the electrostatic interactions between the negatively charged PLGA and positively charged CS, indicating adsorption of CS onto the surface of the PLGA NP [11]. PLGA NPs formulated without CS (Table 1, Samples 5.1 and 6.1) were negatively charged, as consistent with the literature [15].

\subsection{Stability Evaluation}

3.2.1. Stability of Prepared Single and Dual Loaded NPs. A major area of concern for NPs is their long-term physical and chemical stability [30]. Long-term stability is generally defined to be at least 12 months [31]. To advance an ARV NP formulation towards clinical usage, the NP and incorporated ARV must be stable. Furthermore, given the limited resources available in developing countries, storage at room temperature is highly desirable. In this study, dual loaded NPs stored at room temperature showed little to no change in morphology (Figure 1(c)), size, PDI (Figure 3(a)), or zeta potential after one year. This stability was also observed for both PLGA-CS-NVP NPs (Figure 3(b)) and PLGA-CS-LMV NPs (data not shown).

This stability of NPs may be due in part to using PVA as a surfactant. Stabilizers such as PVA have been shown to enhance short-term and long-term stability of NP formulations [32]. PVA can act as a stabilizer, providing sufficient steric hindrance between NPs, allowing them to overcome colloidal instabilities. Moreover, PVA may act as a stabilizer by adsorbing to the NP surface, forming an outer layer that is not easily removed during washing.

In this study, NPs stored at $-70^{\circ} \mathrm{C}$ for one year were larger in size with a mean of $500 \mathrm{~nm}$ using DLS and $200 \mathrm{~nm}$ using TEM (Figure 1(e)). However, PDI and zeta potential showed no marked differences upon storage at $-70^{\circ} \mathrm{C}$ for one year (not shown). Between $-70^{\circ} \mathrm{C}$ and $0^{\circ} \mathrm{C}$, freezing and nonfreezing water molecules bind to polymer molecules through hydrogen bonds [33]. Hydrogen bond interactions between the $\mathrm{OH}$ and $\mathrm{NH}_{2}$ groups of chitosan and the $\mathrm{OH}$ groups of PVA may cause the NPs to aggregate, resulting in an apparent increase in NP size [34].

3.2.2. Stability of Lyophilized NPs. Lyophilization has been shown to enhance long-term stability of colloidal NPs [30]. It has been reported that NPs prepared with 2.5-5\% PVA may be lyophilized without the use of a cryoprotectant [30]. However, following washing to remove excess ARV, CS, and PVA, the addition of a cryoprotectant such as sucrose may be required to ensure stability. NPs lyophilized with $10 \%$ sucrose readily dispersed in water upon reconstitution both immediately after lyophilization and following storage at $4^{\circ} \mathrm{C}$ for one year. The presence of an easily dispersible lyophilized powder and conservation of NP size (Figures 3(g) and 3(h)) indicate successful lyophilization of the NPs [30]. At one year, the capsule morphology (Figure 1(e)), size, PDI (Figure 3(c)), and surface charge of NPs lyophilized with sucrose were preserved. These results are similar to changes in size when PLGA NPs lyophilized with 1-3\% sucrose were stored at $4^{\circ} \mathrm{C}$ and $25^{\circ} \mathrm{C}$ for 3 months [35]. However, the size and PDI of dual loaded NPs lyophilized in the absence of sucrose increased when stored at $4^{\circ} \mathrm{C}$ for one year (Figure $3(\mathrm{~d})$ ). This change in size and PDI was also observed for single loaded PLGA-CSLMV NPs lyophilized in the absence of sucrose (Figure 3(e)). These results demonstrate the importance of using sucrose as a cryoprotectant. Finally, nonlyophilized NPs in 10\% sucrose stored at $-20^{\circ} \mathrm{C}$ for one year had no notable differences in morphology (Figure 1(f)), size, or PDI (Figure 3(f)) when compared to NPs directly following their fabrication.

3.3. In Vitro ARV Release Kinetics and Mechanism. Prolonged release of ARVs from polymeric NPs has been reported [36]. However, in vivo ARV release and drug pharmacokinetics may differ from in vitro results [37]. In particular, lysosomes and proteasomes play an important role in the destruction of HIV-1 during endocytosis of the virus. Apart from HIV being internalized by fusion to the cell membrane, HIV can also enter the cell via endocytosis if spared from degradation in late endosomes and lysosomes [38]. If the HIV pathogen remains intact and viable in the endosome, the cell becomes prone to viral infection [39]. Although the internalization of HIV by endocytosis does not result in productive infection [38], it has been reported that infectivity of HIV-1 is enhanced by the inhibition of lysosomal function [39]. In order to predict future biological efficacy, this study sought to determine the drug release kinetics and possible mechanism of LMV and NVP from single or dual loaded NPs. Release studies were performed in phosphate buffer at $37^{\circ} \mathrm{C}$ at $\mathrm{pH} 7.2$ and $\mathrm{pH} 5$ in order to mimic physiologic and late stage lysosomal $\mathrm{pH}$, respectively [40]. At $37^{\circ} \mathrm{C}$ in pH 7.2 (Figure 4), both LMV and NVP in the dual loaded NPs exhibited a biphasic release profile. An initial burst in the first 20 hours was observed for LMV followed by a slow and almost stable release over the remaining 100 hours. Concurrently, within the first 20 hours, NVP was slowly released initially but gradually surpassed LMV levels over the remaining 100 hours.

ARV release in various storage conditions was determined (Figures $4-7$ ). At $4^{\circ} \mathrm{C}$, there was no significant initial burst release of LMV. For both ARVs, consistent but lower drug amounts were released (Figure 5) compared to that at $37^{\circ} \mathrm{C}$. At $4^{\circ} \mathrm{C}$, water absorption and hydration of PLGA and/or chitosan within the NP may have decreased. This resulted in reduced NP degradation and subsequently less LMV being released.

During NP fabrication, ARVs may be adsorbed to the NP surface, entrapped within the NP, or chemically conjugated to the NP [41], or the NP may be a nanocrystal of the ARV itself [42]. Release of ARVs from PLGA NPs is initially controlled by diffusion, whereas in the final stages ARV release is controlled by dissolution/erosion [43, 44]. The release of NVP is primarily dependent on the degradation of the PLGA core and NVP's poor water solubility. Additionally, ionic interactions between NVP and PLGA may result in shielding PLGA's terminal carboxyl group. This shielding reduces the hydrophilicity of the polymer, thereby lessening the PLGA erosion and its concomitant NVP release [45]. The observed initial burst release of LMV suggests that once the 


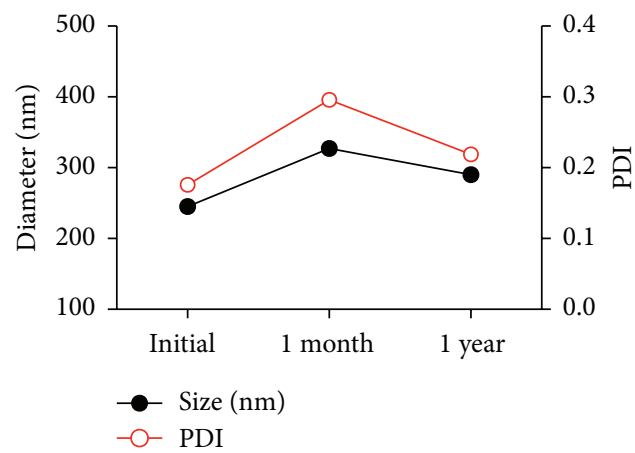

(a)

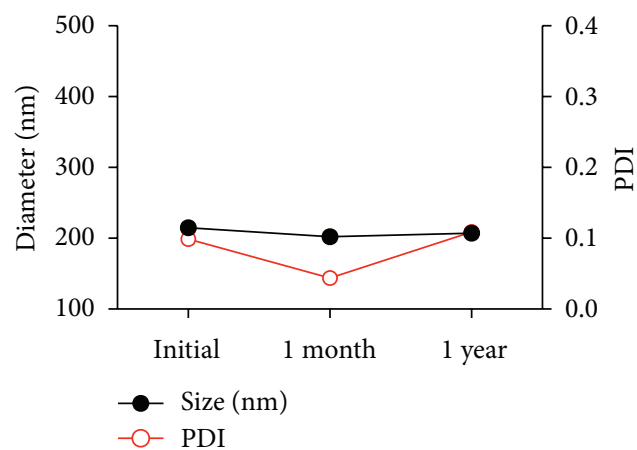

(c)

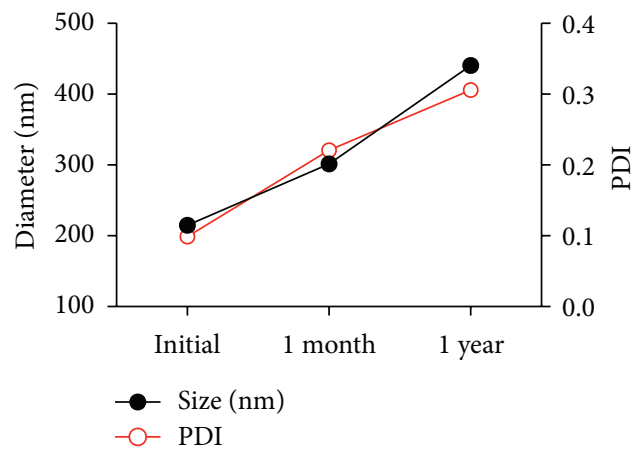

(e)

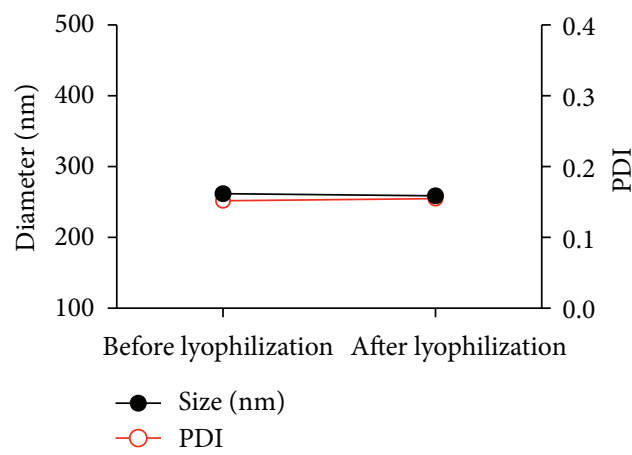

$(\mathrm{g})$

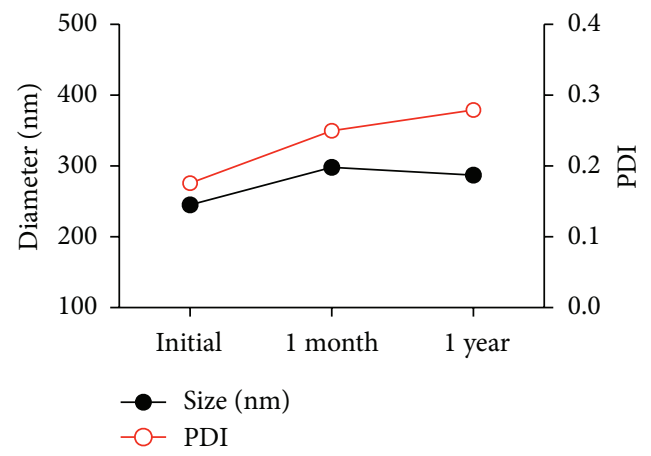

(b)

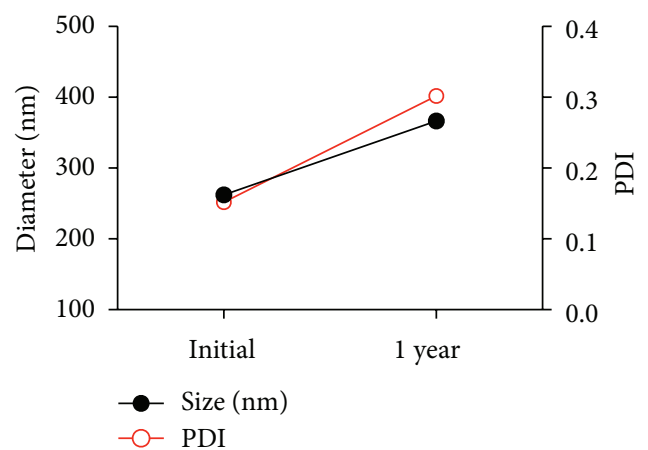

(d)

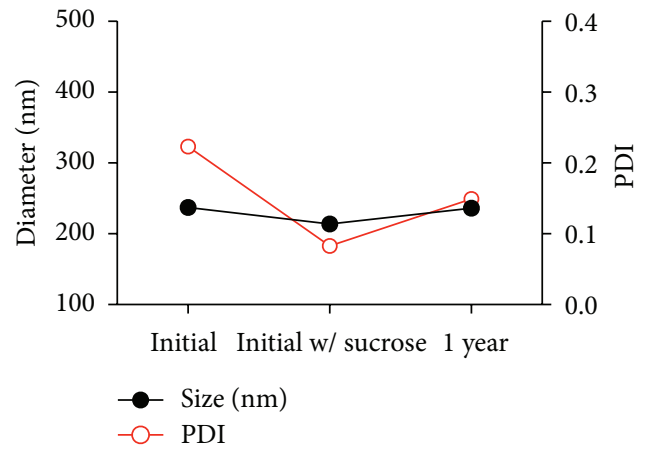

(f)

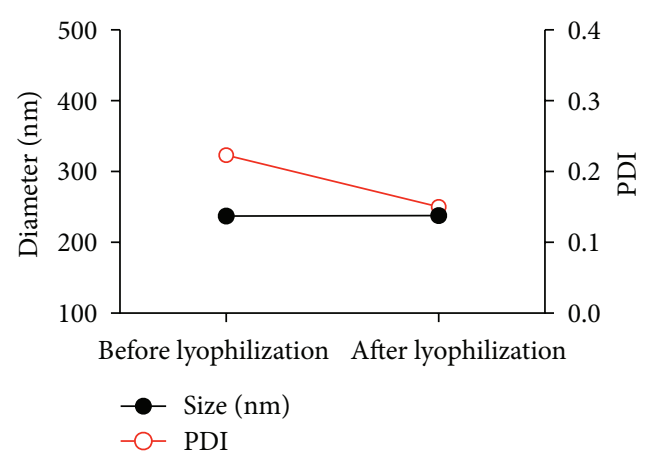

(h)

FIGURE 3: Size and PDI stability of NPs at different storage conditions; (a), (b), (c), and (f) were stable while (d) and (e) were unstable. Size is maintained after lyophilization (g and h). (a) PLGA-CS-NVP-LMV NPs stored at room temperature; (b) PLGA-CS-NVP NPs stored at room temperature; (c) PLGA-CS-LMV NPs lyophilized with $10 \%$ sucrose and stored at $4^{\circ} \mathrm{C}$; (d) PLGA-CS-NVP-LMV NPs lyophilized without sucrose and stored at $4^{\circ} \mathrm{C}$; (e) PLGA-CS-LMV NPs lyophilized without sucrose and stored at $4^{\circ} \mathrm{C}$; (f) PLGA-CS-NVP NPs in $10 \%$ sucrose stored at $-20^{\circ} \mathrm{C}$; (g) PLGA-CS-NVP-LMV NPs lyophilized in 10\% sucrose; (h) PLGA-CS-NVP NPs lyophilized in 10\% sucrose. 


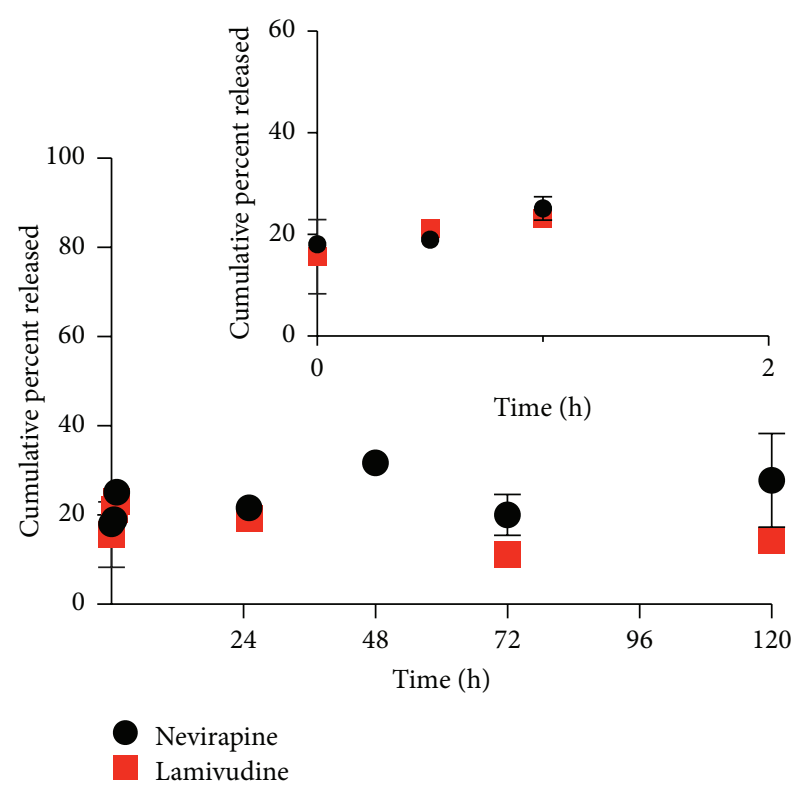

Figure 4: Release of LMV and NVP from PLGA-CS-NVP-LMV NPs at $37^{\circ} \mathrm{C}$ in $\mathrm{PBS}$ pH 7.2 (mean $n=3$ ).

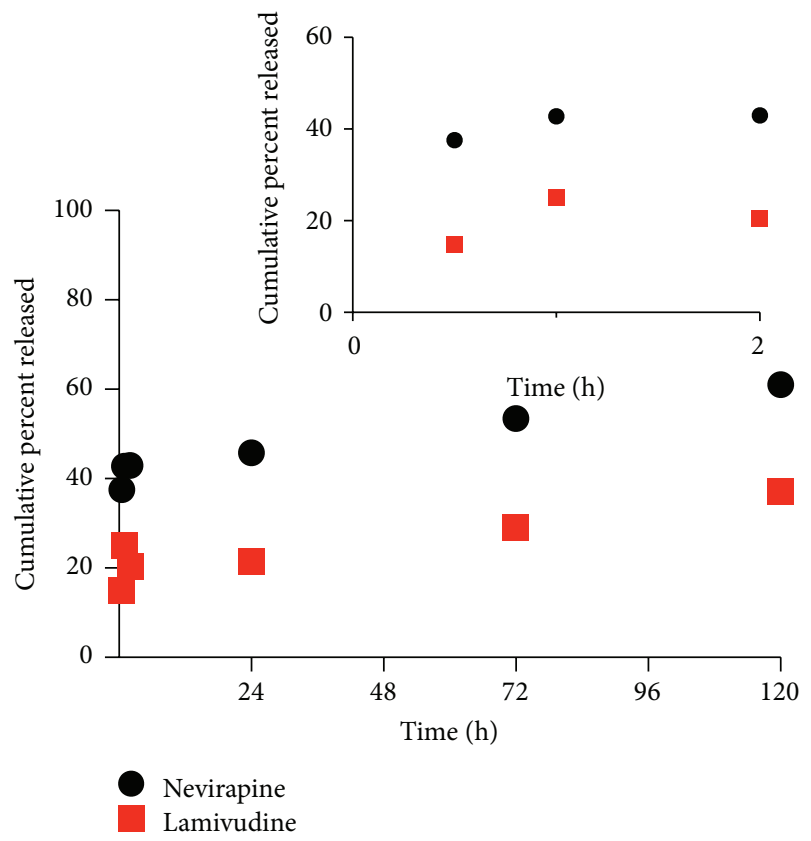

FIGURE 5: Release of LMV and NVP from PLGA-CS-NVP-LMV NPs at $4^{\circ} \mathrm{C}$ in PBS pH 7.2 (mean $\left.n=3\right)$.

hydrophobic PLGA core is exposed and perhaps partially disrupted, a portion of the LMV is immediately physically released and quickly diffuses through the outer, hydrophilic CS shell, although some LMV may eventually be adsorbed on the outer surface [46]. The slower, later release of LMV could result from the rest of the LMV that is entrapped within the PLGA core being more slowly released as the PLGA core undergoes less rapid dissolution.

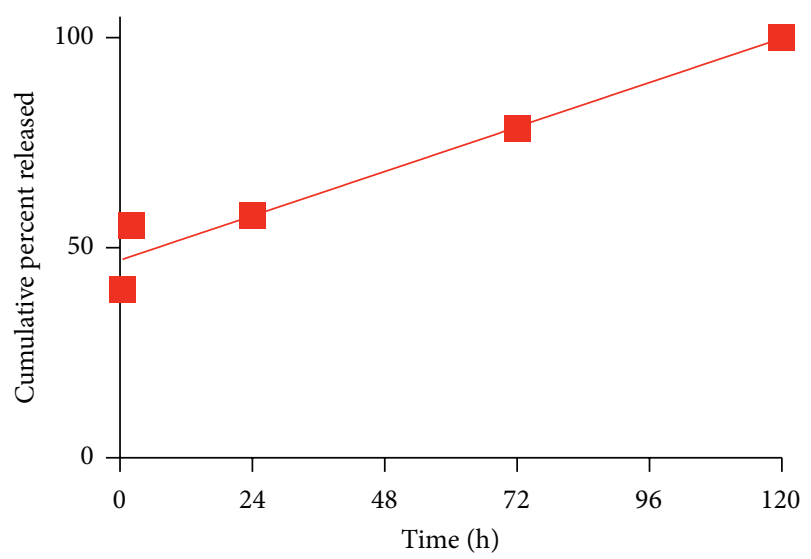

FIGURE 6: Release kinetics of PLGA-CS-LMV NPs in $\mathrm{pH} 5$ at $37^{\circ} \mathrm{C}$. Data are fit using the zero-order model.

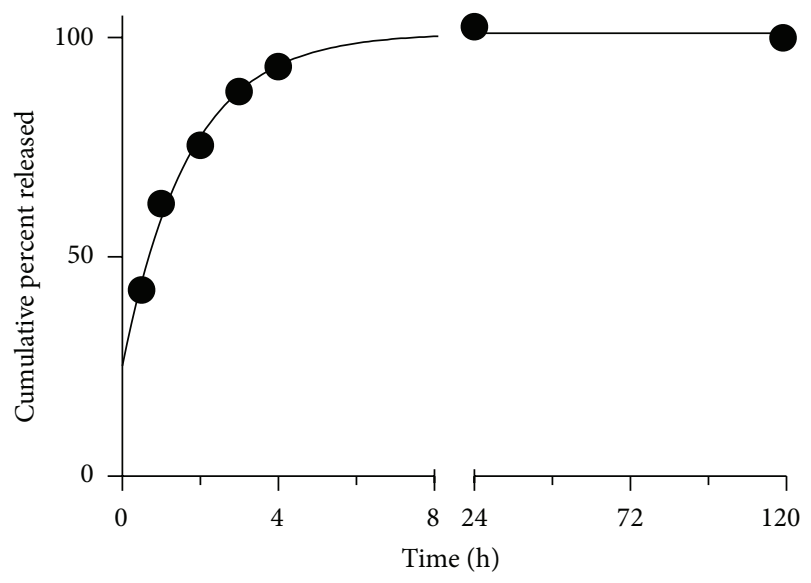

FIGURE 7: Release kinetics of PLGA-CS-NVP NPs in $\mathrm{pH} 7.2$ at $37^{\circ} \mathrm{C}$. Data are fit using the first-order model.

Based on the best fit regression model, NVP exhibited a first-order release mechanism in $\mathrm{pH} 7.2$ (Figure 7). The release of LMV in pH 5 was determined to be zero order (Figure 6). At this $\mathrm{pH}$, late lysosomes and endosomes play an important role in the degradation of foreign matter in the endocytotic pathway [38]. In the presence of lysosomotropic agents in HIV patients, the release profile of LMV may play an important role in derailing HIV infectivity.

\section{Conclusion}

We have successfully fabricated positively charged, biodegradable PLGA-CS NPs that integrate both a hydrophobic and a hydrophilic ARV using a reproducible method. ARV release from a dual loaded NP, where one drug is released as a burst before the other, can be of therapeutic significance in HIV management. The initial LMV burst followed by a decrease that is paralleled by an increase in NVP observed in PBS pH 7.2 at $37^{\circ} \mathrm{C}$ may entail efficacy in inhibiting enzymes targeted by these ARVs at different times. This phenomenon may go a long way in managing viral load in 
the plasma and possibly prolong the ability of the HIV virus to develop resistance. The stability of these core-shell NPs demonstrates the ability to reformulate two first-generation ARV s into a nanoformulation with more favorable properties. In resource-limited settings, the stability of ARV-loaded NPs at room temperature would reduce expenses associated with cold-chain logistics and distribution, allowing for increased access to these medications. The observed stability of nonlyophilized NPs with 10\% sucrose may be another storage strategy where lyophilization equipment may not be readily available. Our approach to fabricating dual loaded NPs, with stable physicochemical properties, offers the potential of further developing novel nanoformulations for use in HIV1 infection. Further investigation of these core-shell ARV nanomedicines, using cellular and animal models of HIV-1 infection, will provide information critical to their clinical advancement.

\section{Disclosure}

Part of the work in this paper was presented under "posters category" at the Conference on Retroviruses and Opportunistic Infections (CROI) 2015, Seattle, Washington. The content presented in this paper is solely the responsibility of the authors and does not necessarily represent the official views of the Fogarty International Center, National Institute of Allergy and Infectious Diseases, or the National Institutes of Health.

\section{Competing Interests}

The authors report no competing interests in this work.

\section{Authors' Contributions}

Faithful Makita-Chingombe and Hilliard L. Kutscher contributed equally.

\section{Acknowledgments}

This project was supported in part by Grants U01AI068636 and HHSN272201500006C from the National Institutes of Health and National Institute of Allergy and Infectious Diseases (NIAID); Grant D43TW007991 from the National Institutes of Health and Fogarty International Center AIDS International Training and Research and Grant (AITRP); and the University of Rochester Center for AIDS Research Grant P30AI078498 (NIH/NIAID) and the University of Rochester School of Medicine and Dentistry. Hilliard L. Kutscher is supported by Ruth L. Kirschstein National Research Service Award (NRSA) Institutional Research Training Grant 1T32GM099607. The collaborative contributions and dedication of the research staff from the Translational Pharmacology Research Core at the University at Buffalo are appreciated. The authors would also like to express their appreciation to Waters Corporation for their generous donation of an HPLC.

\section{References}

[1] World Health Organization, "HIV/AIDS Fact Sheet," http:// www.who.int/mediacentre/factsheets/fs360/en/.

[2] UNAIDS, "How AIDS changed everything-MDG6: 15 years, 15 lessons of hope from the AIDS response," http://www.unaids .org/en/resources/documents/2015/MDG6_15years-15lessonsfromtheAIDSresponse.

[3] World Health Organization, Consolidated Guidelines on the Use of Antiretroviral Drugs for Treating and Preventing HIV Infection, World Health Organization, Geneva, Switzerland, 2013, http//www.who.int/hiv/pub/guidelines/arv2013/download/ en/.

[4] National Institutes of Health, "Guidelines for the use of antiretroviral agents in HIV-1-infected adults and adolescents," https://aidsinfo.nih.gov/guidelines/html/1/adult-and-adolescenttreatment-guidelines/0.

[5] S. Mitra, U. Gaur, P. C. Ghosh, and A. N. Maitra, "Tumour targeted delivery of encapsulated dextran-doxorubicin conjugate using chitosan nanoparticles as carrier," Journal of Controlled Release, vol. 74, no. 1-3, pp. 317-323, 2001.

[6] E. Miele, G. P. Spinelli, E. Miele, F. Tomao, and S. Tomao, "Albumin-bound formulation of paclitaxel (Abraxane ABI-007) in the treatment of breast cancer," International Journal of Nanomedicine, vol. 4, pp. 99-105, 2009.

[7] F. Danhier, E. Ansorena, J. M. Silva, R. Coco, A. Le Breton, and V. Préat, "PLGA-based nanoparticles: an overview of biomedical applications," Journal of Controlled Release, vol. 161, no. 2, pp. 505-522, 2012.

[8] S. D. Mahajan, I. Roy, G. Xu et al., "Enhancing the delivery of anti retroviral drug 'saquinavir' across the blood brain barrier using nanoparticles," Current HIV Research, vol. 8, no. 5, pp. 396-404, 2010.

[9] M. I. Alam, S. Beg, A. Samad et al., "Strategy for effective brain drug delivery," European Journal of Pharmaceutical Sciences, vol. 40, no. 5, pp. 385-403, 2010.

[10] K. Nagpal, S. K. Singh, and D. N. Mishra, "Drug targeting to brain: a systematic approach to study the factors, parameters and approaches for prediction of permeability of drugs across BBB," Expert Opinion on Drug Delivery, vol. 10, no. 7, pp. 927955,2013

[11] A. Dube, J. L. Reynolds, W.-C. Law, C. C. Maponga, P. N. Prasad, and G. D. Morse, "Multimodal nanoparticles that provide immunomodulation and intracellular drug delivery for infectious diseases," Nanomedicine, vol. 10, no. 4, pp. 831-838, 2014.

[12] H. Dai, X. Jiang, G. C. Y. Tan et al., "Chitosan-DNA nanoparticles delivered by intrabiliary infusion enhance liver-targeted gene delivery," International Journal of Nanomedicine, vol. 1, no. 4, pp. 507-522, 2006.

[13] M. Wang, Y. Zhang, J. Feng et al., "Preparation, characterization, and in vitro and in vivo investigation of chitosan-coated poly (d,l-lactide-co-glycolide) nanoparticles for intestinal delivery of exendin-4," International Journal of Nanomedicine, vol. 8, pp. 1141-1154, 2013.

[14] S. H. Bakhru, E. Altiok, C. Highley et al., "Enhanced cellular uptake and long-term retention of chitosan-modified ironoxide nanoparticles for MRI-based cell tracking," International Journal of Nanomedicine, vol. 7, pp. 4613-4623, 2012.

[15] L. Chronopoulou, M. Massimi, M. F. Giardi et al., "Chitosancoated PLGA nanoparticles: a sustained drug release strategy for cell cultures," Colloids and Surfaces B: Biointerfaces, vol. 103, pp. 310-317, 2013. 
[16] Y. Luo, Z. Teng, Y. Li, and Q. Wang, "Solid lipid nanoparticles for oral drug delivery: chitosan coating improves stability, controlled delivery, mucoadhesion and cellular uptake," Carbohydrate Polymers, vol. 122, pp. 221-229, 2015.

[17] P. N. Prasad, Introduction to Nanomedicine and Nanobioengineering, John Wiley \& Sons, New York, NY, USA, 2012.

[18] H. L. Kutscher, F. Makita-Chingombe, S. DiTursi et al., "Macrophage targeted nanoparticles for antiretroviral (ARV) delivery," Journal of Personalized NanoMedicine, vol. 1, no. 2, pp. 40-48, 2015.

[19] C. Stigliano, S. Aryal, M. D. de Tullio et al., "siRNA-chitosan complexes in poly(lactic-co-glycolic acid) nanoparticles for the silencing of aquaporin-1 in cancer cells," Molecular Pharmaceutics, vol. 10, no. 8, pp. 3186-3194, 2013.

[20] N. L. Rezk, R. R. Tidwell, and A. D. M. Kashuba, "Simultaneous determination of six HIV nucleoside analogue reverse transcriptase inhibitors and nevirapine by liquid chromatography with ultraviolet absorbance detection," Journal of Chromatography B, vol. 791, no. 1-2, pp. 137-147, 2003.

[21] A. G. Nelson, X. Zhang, U. Ganapathi et al., "Drug delivery strategies and systems for HIV/AIDS pre-exposure prophylaxis and treatment," Journal of Controlled Release, vol. 219, pp. 669680, 2015.

[22] H. L. Kutscher, P. N. Prasad, G. D. Morse, and J. L. Reynolds, "Emerging nanomedicine approaches to targeting HIV-1 and antiretroviral therapy," Future Virology, vol. 11, no. 2, pp. 101-104, 2016.

[23] B. J. Edagwa, T. Zhou, J. M. McMillan, X.-M. Liu, and H. E. Gendelman, "Development of HIV reservoir targeted long acting nanoformulated antiretroviral therapies," Current Medicinal Chemistry, vol. 21, no. 36, pp. 4186-4198, 2014.

[24] A. Lamprecht, N. Ubrich, M. Hombreiro Pérez, C.-M. Lehr, M. Hoffman, and P. Maincent, "Biodegradable monodispersed nanoparticles prepared by pressure homogenizationemulsification," International Journal of Pharmaceutics, vol. 184, no. 1, pp. 97-105, 1999.

[25] E. Piñón-Segundo, M. G. Nava-Arzaluz, and D. LechugaBallesteros, "Pharmaceutical polymeric nanoparticles prepared by the double emulsion-solvent evaporation technique," Recent Patents on Drug Delivery and Formulation, vol. 6, no. 3, pp. 224235, 2012.

[26] nanoComposix, "nanoComposix' guide to dynamic light scattering measurement and analysis," 2015, https://cdn.shopify .com/s/files/1/0257/8237/files/nanoComposix_Guidelines_for_ DLS_Measurements_and_Analysis.pdf.

[27] http://www.nbip.dcu.ie/papers/DLS.pdf.

[28] H. Cui, Y. Feng, W. Ren, T. Zeng, H. Lv, and Y. Pan, "Strategies of large scale synthesis of monodisperse nanoparticles," Recent Patents on Nanotechnology, vol. 3, no. 1, pp. 32-41, 2009.

[29] T. Sugimoto, Monodispersed Particles, 2001.

[30] W. Abdelwahed, G. Degobert, S. Stainmesse, and H. Fessi, "Freeze-drying of nanoparticles: formulation, process and storage considerations," Advanced Drug Delivery Reviews, vol. 58, no. 15, pp. 1688-1713, 2006.

[31] M. H. Golden, D. C. Cooper, M. T. Riebe, and K. E. Carswell, "A matrixed approach to long-term stability testing of pharmaceutical products," Journal of Pharmaceutical Sciences, vol. 85, no. 2, pp. 240-245, 1996.

[32] L. Wu, J. Zhang, and W. Watanabe, "Physical and chemical stability of drug nanoparticles," Advanced Drug Delivery Reviews, vol. 63, no. 6, pp. 456-469, 2011.
[33] S. J. Kim, S. J. Park, and S. I. Kim, "Swelling behavior of interpenetrating polymer network hydrogels composed of poly(vinyl alcohol) and chitosan," Reactive and Functional Polymers, vol. 55, no. 1, pp. 53-59, 2003.

[34] K. Yao, J. Li, F. Yao, and Y. Yin, Chitosan-Based Hydrogels: Functions and Applications, CRC Press, 2011.

[35] M. Holzer, V. Vogel, W. Mäntele, D. Schwartz, W. Haase, and K. Langer, "Physico-chemical characterisation of PLGA nanoparticles after freeze-drying and storage," European Journal of Pharmaceutics and Biopharmaceutics, vol. 72, no. 2, pp. 428437, 2009.

[36] N. M. Khalil, E. Carraro, L. F. Cótica, and R. M. Mainardes, "Potential of polymeric nanoparticles in AIDS treatment and prevention," Expert Opinion on Drug Delivery, vol. 8, no. 1, pp. 95-112, 2011.

[37] S. Dash, P. N. Murthy, L. Nath, and P. Chowdhury, "Kinetic modeling on drug release from controlled drug delivery systems," Acta Poloniae Pharmaceutica, vol. 67, no. 3, pp. 217-223, 2010.

[38] B. L. Fredericksen, B. L. Wei, J. Yao, T. Luo, and J. V. Garcia, "Inhibition of endosomal/lysosomal degradation increases the infectivity of human immunodeficiency virus," Journal of Virology, vol. 76, no. 22, pp. 11440-11446, 2002.

[39] B. L. Wei, P. W. Denton, E. O’Neill, T. Luo, J. L. Foster, and J. V. Garcia, "Inhibition of lysosome and proteasome function enhances human immunodeficiency virus type 1 infection," Journal of Virology, vol. 79, no. 9, pp. 5705-5712, 2005.

[40] A. Sorkin and M. von Zastrow, "Signal transduction and endocytosis: close encounters of many kinds," Nature Reviews Molecular Cell Biology, vol. 3, no. 8, pp. 600-614, 2002.

[41] K. S. Soppimath, T. M. Aminabhavi, A. R. Kulkarni, and W. E. Rudzinski, "Biodegradable polymeric nanoparticles as drug delivery devices," Journal of Controlled Release, vol. 70, no. 1-2, pp. 1-20, 2001.

[42] S. Balkundi, A. S. Nowacek, R. S. Veerubhotla et al., "Comparative manufacture and cell-based delivery of antiretroviral nanoformulations," International Journal of Nanomedicine, vol. 6, pp. 3393-3404, 2011.

[43] S. Fredenberg, M. Wahlgren, M. Reslow, and A. Axelsson, "The mechanisms of drug release in poly(lactic-co-glycolic acid)based drug delivery systems-a review," International Journal of Pharmaceutics, vol. 415, no. 1-2, pp. 34-52, 2011.

[44] M. Körber, "PLGA erosion: solubility- or diffusion-controlled?" Pharmaceutical Research, vol. 27, no. 11, pp. 2414-2420, 2010.

[45] M. Miyajima, A. Koshika, J. I. Okada, A. Kusai, and M. Ikeda, "The effects of drug physico-chemical properties on release from copoly (lactic/glycolic acid) matrix," International Journal of Pharmaceutics, vol. 169, no. 2, pp. 255-263, 1998.

[46] B. Wang, G. Chen, Z. Mao, Y. Zhang, D. Yu, and C. Gao, "Preparation and cellular uptake of PLGA particles loaded with lamivudine," Chinese Science Bulletin, vol. 57, no. 31, pp. 39853993, 2012. 

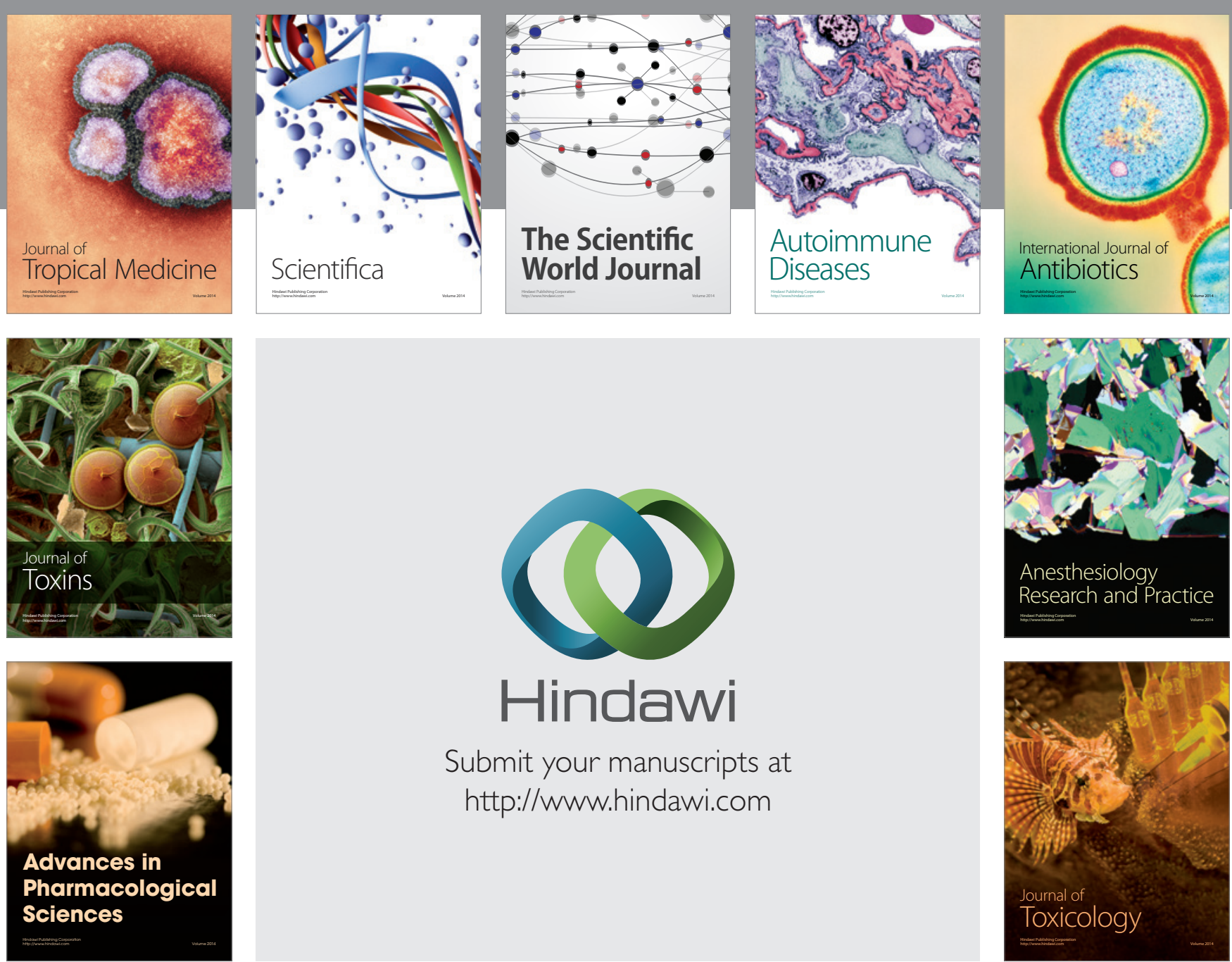

\section{Hindawi}

Submit your manuscripts at

http://www.hindawi.com
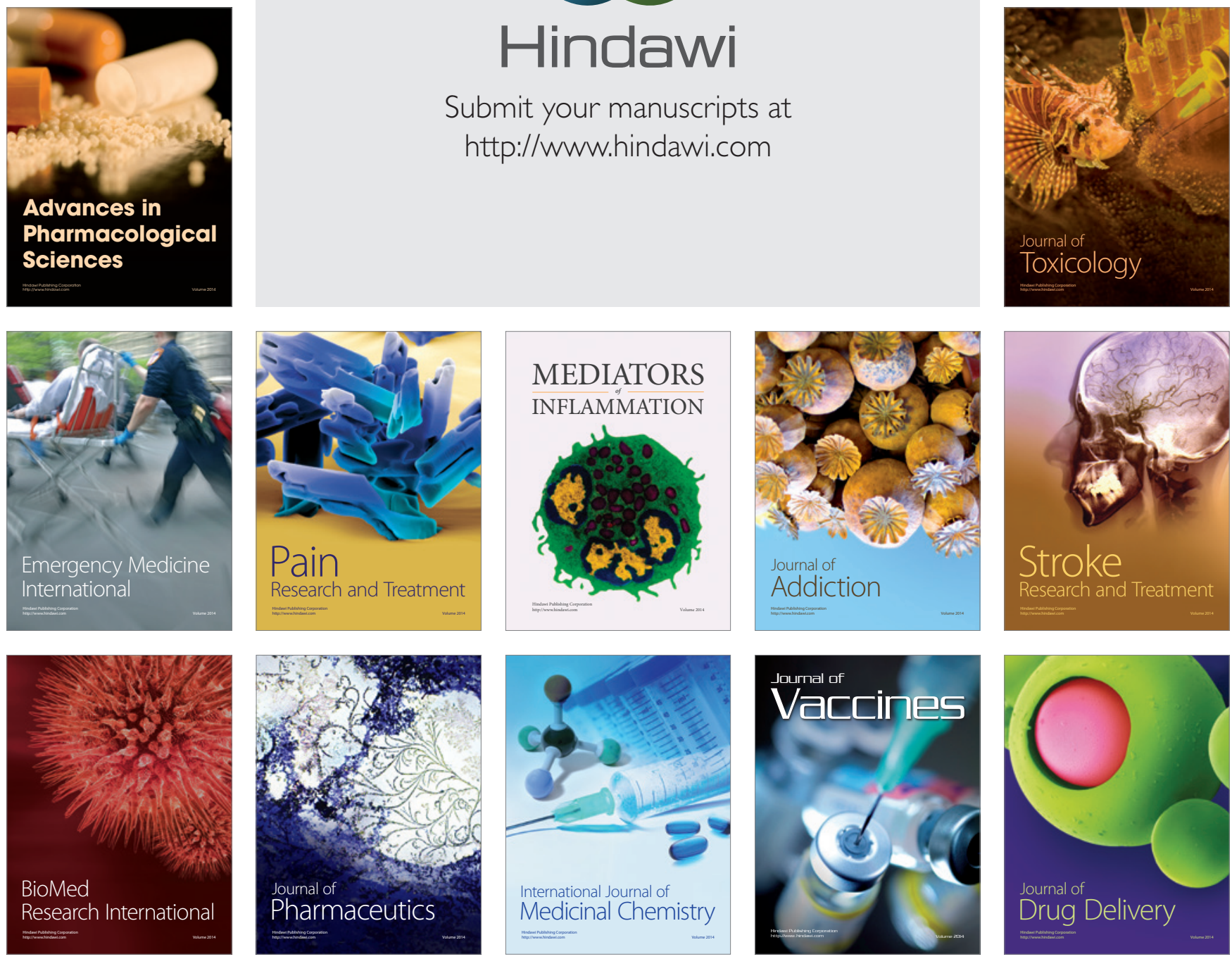